(heartburn 2\% $\mathrm{p}=0.172$, acid regurgitation $4 \% \mathrm{p}=0.31$ ) but significantly lower (better) than GORD participants (heartburn $31 \% \mathrm{p}=<0.001$, acid regurgitation $25 \% \mathrm{p}=0.001)$. NDBO participants with moderate/severe GORD symptoms were associated with higher rates of anxiety $(p=<0.001)$, depression $(p=<0.001)$ and cancer specific worry $(p=<0.001)$. Those who correctly perceived their cancer risk as low tended to have significantly lower rates of cancer worry $(p=<0.001)$.

Conclusions This study provides a valuable insight into the problems BO patients may face. Based on these findings future care pathways must be more patient focused with greater reassurance and communication to address misconceptions of cancer risk, oesophageal cancer related worry and GORD symptom control.

\section{PTU-049 RFA FOR BARRETT'S LOW GRADE DYSPLASIA: RESULTS OF THE 1ST COHORT TREATED IN EAST MIDLANDS}

\begin{abstract}
1,2Ana Victoria García-Marín*, 1,2Jacobo Ortiz-Fernández-Sordo, 1,2Jonathan White, 1,2Jose Santiago-García, ${ }^{3}$ Andy Cole, ${ }^{4}$ John Decastecker, ${ }^{1,2}$ Philip Kaye, ${ }^{1,2}$ Krish Ragunath. ${ }^{1}$ Nottingham Digestive Diseases Centre, University of Nottingham, Nottingham, UK; ${ }^{2}$ NIHR $B R C$ at Nottingham University Hospitals and University of Nottingham, Nottingham, UK; ${ }^{3}$ Leicester General Hospital, UK; ${ }^{4}$ Royal Derby Hospital, UK
\end{abstract}

\subsection{6/gutjnl-2019-BSGAbstracts.262}

Introduction Endoscopic treatment with radiofrequency ablation (RFA) is the ablative therapy of choice for patients with flat high-grade dysplasia (HGD) and residual Barrett's oesophagus (BO) after endoscopic mucosal resection (EMR) of visible lesions. The latest BSG guidelines have recommended it for low-grade dysplasia (LGD) as well. We aim to assess safety and effectiveness from the East Midlands Barrett's RFA database.

Methods Analysis of a prospectively collected database was performed. All patients who underwent RFA for confirmed LGD on two separate occasions between February 2011 and June 2018 were included for analysis. RFA was performed with either the balloon or focal device or both, depending on Barrett's length and preferences of the endoscopist. All patients were maintained on high dose twice daily acid suppression with proton pump inhibitors and $\mathrm{H} 2$ antagonists for 2 weeks immediate post RFA. Sessions were performed every 3 months until the BO was endoscopically eradicated. Confirmed remission of $\mathrm{BO}$ was defined as the absence of dysplasia (CRD) and intestinal metaplasia (CRIM) in all the biopsies taken from the GOJ and original BO length at follow up endoscopy 3 months after the RFA session.

Results A total of 41 patients were included for analysis $(88 \%$ male), median age 70 years (mean 66.9). Mean number of RFA sessions per patient was 3.2 (2-6). Average duration of treatment was 38.5 weeks (10-84). 10/41 patients are still undergoing treatment, 28/41 completed RFA protocol and the treatment was discontinued in 3 patients due to histological progression ( 2 cases) or complication (1 case, complex stricture). $90.3 \%$ (28/31) of patients achieved CRD and CRIM at first follow up endoscopy.

During RFA treatment, histological progression occurred in 6 patients (14.6\%): HGD $=4$ and adenocarcinoma=2. Treatment for HGD was EMR followed by RFA in 3 and RFA alone in 1 patient without visible lesion. The 2 patients with adenocarcinoma required curative surgery.

Most common complication requiring intervention was oesophageal stricture in $17.1 \%$ (7/41 patients). All successfully managed with endoscopic dilatation but RFA was abandoned in 1 patient.

Conclusions RFA for LGD is safe and has a high success rate. However, a higher incidence of post RFA strictures was observed in this study that requires investigating. Progression to HGD or cancer was seen in almost $15 \%$ of patients, which highlights the importance of offering treatment to this cohort of Barrett's patients.

\section{PTU-050 MODIFIABLE RISK FACTORS FOR GASTRO-OESOPHAGAL REFLUX DISEASE: A MENDELIAN RANDOMISATION STUDY}

1,2 Harry Green*, ${ }^{1}$ Robin Beaumont, ${ }^{1}$ Samuel Jones, ${ }^{1}$ Hanieh Yaghootkar, ${ }^{1}$ Andrew Wood ${ }^{2}$ James Goodhand, ${ }^{2}$ Nicholas Kennedy, ${ }^{2}$ Tariq Ahmad, ${ }^{1}$ Timothy Frayling, 'Michael Weedon, ${ }^{1}$ Jessica Tyrrell. ${ }^{1}$ University of Exeter, Exeter, UK; ${ }^{2}$ Royal Devon and Exeter NHS Foundation Trust, Exeter, UK

\subsection{6/gutjnl-2019-BSGAbstracts.263}

Introduction Gastro-oesophageal Reflux Disease (GORD) is a common condition in which acid from the stomach leaks to the oesophagus causing heartburn and unpleasant taste in mouth, potentially leading to Barrett's Oesophagus and Oesophageal Adenocarcinoma. Observationally, it has been reported that low body mass index (BMI), high BMI, past smoking, alcohol and caffeine consumption increase risk of GORD. Recently, it was reported that waist hip ratio (WHR) was a better measure of obesity than BMI for predicting GORD. However, observational relationships may be affected by bias and confounding.

Methods We used data from 379,713 unrelated European participants in the UK Biobank, including 23,123 GORD cases to firstly explore the observational associations between GORD and adiposity measures, smoking status and smoking frequency, alcohol and caffeine consumption.

We then used one- and two-sample Mendelian Randomisation (MR) techniques to test the causal relationship between the predictors and GORD. MR is a genetic technique which uses genetic variation to examine the causal effect of a risk factor on an outcome through the use of a genetic instrument for the risk factor.

Results Observationally male gender, age, past smoking, current smoking, higher BMI, higher WHR, higher body fat percentage and waist circumference associated with higher odds of GORD, whilst higher caffeinated coffee consumption was associated with lower risk of GORD. There was some tentative evidence of a J-shaped relationship between BMI and GORD. Alcohol consumption was not associated with GORD.

MR provided strong evidence of a causal role for WHR, with a one-SD higher WHR causing a 1.34 higher odds of GORD (95\%CI: 1.16-1.55, $p=5 e-5)$. Similarly, a one-SD higher WHR adjusted for BMI was associated with 1.21 higher odds of GORD (95\%CI: 1.14-1.29, $p=2 e-9$ ). However, there was no evidence of a causal relationship for BMI (OR: 1.00 (95\%CI: 0.09-1.12), $p=0.97$ ). There was no evidence of a causal role for body fat percentage, smoking and caffeine. All results were robust to 2 sample MR approaches.

Conclusions The results show robust causal evidence for the link between obesity and reflux being primarily down to WHR, not BMI. These results emphasise the importance of measuring WHR when studying gastrointestinal disorders, and the importance of weight loss in reducing the risk of reflux. 